\title{
Demonstrativos, determinantes e definitude em Wapichana
}

\author{
Marcelo Giovannetti \\ Renato Miguel Basso*
}

Resumo

O Wapichana é uma língua amazônica pertencente à família Arawak, apresentando entre 6.000 e 7.000 falantes, nos territórios de Roraima (Brasil), Venezuela e Guiana. As línguas Arawak são conhecidas por não apresentarem um sistema complexo de determinantes, e normalmente não apresentam artigos definidos, embora haja demonstrativos (cf. SANTOS, 2006). Neste artigo, analisamos dois demonstrativos da língua, wyry'y e diura'a, respectivamente, proximal e distal, e defenderemos que um terceiro item, taury'y, também tido como demonstrativo, não se comporta como um demonstrativo propriamente dito, sendo, na verdade, responsável por traços característicos de definitude semântica, de modo que ele está passando por um processo de gramaticalização. Para tanto, este artigo apresentará as seguintes seções: na primeira seção, apresentaremos algumas características tipológicas do Wapichana; na segunda seção, caracterizaremos a estrutura funcional do DP na língua, mostrando que esta língua apresenta apenas demonstrativos, possessivos e, como argumentaremos, uma espécie de artigo. Na terceira seção, discutiremos as características da definitude e dos demonstrativos (DIESSEL, 1999), e argumentaremos que 'taury'y' não se comporta como um. Na seção quatro, discutiremos brevemente sobre o fenômeno da gramaticalização para, na seção cinco, apresentarmos nossa análise sobre o item "taury'y". Finalizaremos este artigo mostrando dados da língua que atestam nossas hipóteses sobre 'taury'y' no Wapichana estar passando por um processo de gramaticalização.

Palavras-chave: Artigo. Definitude. Demonstrativo. Gramaticalização. Relativizador. Wapichana.

\footnotetext{
* Universidade Federal de São Carlos / CNPq.
} 
Wapishana is an Amazonian language which belongs to the Arawakan branch, and is spoken by 6,000 to 7,000 people in the territories of Roraima (Brazil), Venezuela, and Guyana. Arawakan languages are known for not having a complex determiner system, and normally they lack definite articles but have demonstratives (cf. SANTOS, 2006). In this paper, we analyze two Wapishana demonstratives, 'wyry'y' and 'diura'a', respectively a proximal and a distal one, and argue that a third item, 'taury'y', usually also considered a demonstrative, is in fact a definite article. We argue that 'taury'y' does not behave as a proper demonstrative, but it is responsible for the feature related to the semantic definiteness, and it is going through a process of grammaticalization. This paper is organized as the following: in the first section, we present some Wapishana typological features; in the second section, we characterize the functional structure of DP in the language, showing that Wapishana presents only demonstratives, possessives and as we'll argue a sort of article. In the third section, we discuss the features of definiteness and demonstratives (DIESSEL, 1999), and argue that 'taury'y' does not behave as a demonstrative. In the fourth section, we briefly discuss the phenomenon of grammaticalization; in the fifth section we show our analysis of 'taury'y'. We end up this paper showing some data which support our hypothesis about 'taury'y' in Wapishana, namely, that is going through grammaticalization process.

Keywords: Article. Definiteness. Demonstrative. Grammaticalization. Relativizer. Wapishana. 


\section{Introdução}

definitude nas línguas naturais é uma característica semântica
relacionada aos DPs, cuja investigação ganhou um grande corpo com
a abordagem de Russell (1905) sobre as descrições definidas e a ideia de exigência de unicidade de seu referente. Em geral, um modo muito utilizado pelas línguas do mundo para garantir a unicidade do referente é o emprego de artigos definidos; contudo, grande parte das línguas existentes não possui um sistema completo de determinantes composto por artigos definidos e indefinidos, deixando a cargo da pragmática, ou de outros elementos linguísticos, a indicação da unicidade do referente.

O Wapichana, língua indígena falada no estado de Roraima (Brasil), na Venezuela e na Guiana, apresenta um sistema nominal nu, ou seja, sem determinantes foneticamente realizados. Contudo, ela apresenta um sistema de demonstrativos composto por basicamente três itens, dois dos quais podem ser considerados propriamente demonstrativos, enquanto um terceiro item passa, atualmente, por um processo de gramaticalização, em direção à formação de um possível artigo definido - uma conhecida rota de gramaticalização, que foi tomada, a partir do latim, por línguas como o Português, o Italiano, o Espanhol, o Francês, etc., e também, partindo do proto-germânico, pelas línguas germânicas, como o Inglês, o Alemão, etc.

Revista Letras,

Curitiba, UfPr, n. 96, pp.423-441, jul./dez. 2017. ISSN 2236-0999 (versão eletrônica) 
Via de regra, gramaticalização envolve mudança semântica, rigidez sintática e perda de material fonético/fonológico. Contudo, o que temos no Wapichana não é um processo que tenha afetado (ainda) o nível fonológico, tampouco o sintático, uma vez que ambos ocorrem no interior do DP, ocupando o núcleo de determinante e podem ou não ter o NP complemento preenchido; contudo, no nível semântico há uma especialização desse suposto demonstrativo relacionado à definitude e à presença ou ausência do referente no contexto de proferimento da sentença em que aparece.

O presente artigo está organizado da seguinte forma: na seção 1, apresentamos alguns aspectos tipológicos e estruturais da língua Wapishana; na seção 2, apresentaremos a estrutura funcional do DP no Wapichana, mostrando que essa língua só possui demonstrativos e possessivos e que estes podem aparecer como morfemas presos ao verbo; na seção 3, apresentaremos as características semânticas dos demonstrativos e da definitude; na seção 4, apresentamos brevemente alguns critérios de gramaticalização para, na seção 5 , discutirmos a hipótese de gramaticalização do item 'taury'y'. Finalizamos, na conclusão, com uma retomada do caminho percorrido e os resultados alcançados sobre o estatuto de 'taury'y' na língua Wapichana.

\section{Aspectos tipológicos da língua Wapichana}

Segundo Migliazza (1985), o termo Wapichana é utilizado para designar os falantes de dois dialetos falados no Brasil e na República Cooperativa da Guiana. Essa língua pertence à família Arawak (RODRIGUES, 1986); Santos (2006), baseando-se por sua vez na classificação de Greenberg (1956 apud SANTOS, 2006), afirma que o Wapichana pertence à família Maipuran, subgrupo do tronco Arawakan, filiado ao supermacrotronco Equatorial, embora tal classificação não seja aceita unanimemente por aqueles que se debruçam sobre a tipologia das línguas naturais. Uma outra classificação, bem mais aceita, é a de Payne (1991 apud SANTOS, 2006), de acordo com a qual a língua Wapichana faz parte da família Maipuran, pertencente ao grupo Northern.

A base para tais classificações são comparações realizadas entre línguas tidas como próximas, muito embora não haja dados adequados em número suficiente para uma comparação com o grau de detalhe desejado; nota-se também a grande extensão territorial abrangida pelas línguas em comparação, o que produz uma diversidade linguística no interior da própria família em questão. De acordo com Aikhenvald (1999, p. 73), a designação TA-Arawak foi empregada inicialmente por von den Steinen (1886 apud SANTOS, 2006) a fim de estabelecer a separação entre as línguas cuja primeira pessoa do singular apresenta o prefixo 'ta-' e aquelas em que o sufixo utilizado para a mesma pessoa é 'nu-'. 
M.

GiovannetTi

\& R. M. Basso

Demonstrativos,

determinantes

e definitude em

Wapichana
Revista Letras,

Curitiba, UfPr, n. 96, pp.423-441,

jul./dez. 2017. ISSN 2236-0999

(versão eletrônica)
Morfologicamente, a língua Wapichana apresenta as categorias gramaticais tradicionalmente tomadas como essenciais, tais como nome, verbo, adjetivo, advérbio, numerais, demonstrativos e pronomes; não obstante, nessa língua não encontramos determinantes do tipo dos artigos, embora apresente numerais e pronomes indefinidos adjungidos ao nome. A distinção morfológica de número e gênero também é produtiva nessa língua, sendo realizadas, respectivamente, por '-nau' e '-aibe'; contudo, há trabalhos (PIRES DE OLIVEIRA; GIOVANNETTI, 2016) que afirmam ser o morfema '-nau' não exatamente um morfema de plural, nos moldes que temos para línguas como o PB e o inglês, mas sim um morfema coletivizador, formando uma espécie de nome coletivo, não um plural morfológico.

De acordo com a classificação tipológica de Greenberg (1966), a língua Wapichana é caracterizada como uma língua SVO, embora apresente algumas características de línguas SOV, tal como a presença de posposições ao invés de preposições; ou seja, a forma como essa língua parametriza a relação núcleocomplemento em relação às adposições põe em xeque o Universal 3 de Greenberg, segundo o qual "línguas com ordem normal SOV são posposicionais". Nessas línguas, o substantivo precederia o adjetivo, comportamento compartilhado pelo Wapichana, que, além disso, apresenta concordância de gênero e número entre adjetivo e substantivo, como discutido em Giovannetti (2016).

No que tange à sua morfologia, a língua Wapichana apresenta algumas características peculiares em relação à aglutinação de morfemas à raiz verbal para indicar tempo, modo e aspecto, comportando-se de modo próximo ao das línguas polissintéticas, embora o fato de colocarmos o Wapichana nessa categoria seja ainda uma questão controversa.

Vejamos, na seção seguinte, um pouco mais sobre a estrutura funcional do DP em Wapichana, para então nos voltarmos aos dados do Wapichana que analisaremos neste texto.

\section{Estrutura funcional em Wapichana}

Os estudos da estrutura funcional do DP originaram-se com Abney (1987), em decorrência da tentativa de estender as projeções lexicais para categorias funcionais. $\mathrm{O}$ trabalho pioneiro de Abney deu início a uma extensiva pesquisa sobre a estrutura funcional do DP, que se mostrou ser bem rica e articulada como as que haviam sido desenvolvidas no nível do IP/TP. Contribuições de Brugè (2002), Giusti (2002) e Scott (2002) partiram dessa possível relação entre as estruturas estendidas de IP/TP e do DP e avançaram com novos e importantes insights para o domínio do DP. De acordo com Cinque (2002), há evidências presentes em uma variedade de línguas que nos levam à conclusão de que sintagmas demonstrativos (“demonstrative phrases") são gerados no especificador 
de uma projeção funcional mais baixa que aquelas alojando sintagmas adjetivais atributivos e imediatamente mais alta que NP (sintagmas nominais). O autor ainda afirma que até mesmo aquelas línguas em que obrigatoriamente o sintagma demonstrativo é alçado para uma posição de especificador mais alta (Spec, DP) apresentam vestígios em uma posição mais baixa, evidenciando o local em que tal sintagma foi gerado.

Em Wapichana, a ordem no interior do DP não se explicita de modo simples, uma vez que, por ser uma língua polissintética, algumas características gramaticais são realizadas morfologicamente, como, por exemplo, a posse, que em $\mathrm{PB}$ é expressa pelos pronomes possessivos, ocupando um núcleo PossP mais alto que o nome e mais baixo que o determinante, podendo gerar sintagmas como "a minha roça”; naquela língua, a posse é realizada por meio de um morfema prefixal e um sufixal "unzakapan" ('minha roça'); quando em sua forma livre ("ungary zakapan” 'minha roça'), o possessivo ocupa o núcleo Poss, deixando o núcleo D vazio. Como o Wapichana não possui artigos, podemos asseverar que o núcleo D é ocupado apenas pelo demonstrativo em uma estrutura do tipo “wyry'y unzakapan” ('essa minha roça'/essa raça é minha'), com o possessivo ocupando o núcleo Poss: [ ${ }_{\mathrm{DP}}$ Wyry'y PossP $_{\text {un- }}$ [NP zakapan]]].

Além da forma presa 'un', o Wapichana também apresenta uma forma livre de possessivo, 'ungary', de modo que o sintagma 'a minha roça' poderia ter a configuração "ungary zakapan” ('minha roça'), e a construção com o demonstrativo, a forma "wyry'y" ungary zakapan" ('essa minha roça'). Isso confirma nossa hipótese de que o Wapichana possui uma estruturação interna do $\mathrm{DP}$ semelhante àquela apresentada pelo $\mathrm{PB}$, no que tange à questão de o 428 determinante ocupar uma posição mais alta na estrutura que o possessivo. Contudo, não podemos afirmar se, naquela língua, o demonstrativo é gerado em uma posição mais baixa na estrutura e movido para [Spec, DP] ou se ele é gerado em uma posição mais alta, permanecendo in situ, uma vez que não encontramos dados em que o demonstrativo apareça em uma posição pós-nominal.

Em relação à posição do adjetivo no interior do sintagma nominal, o Wapichana apresenta tanto a ordem canônica Nome-Adjetivo, como em 'zynaba maydaiaru'u' ("mulher solteira"), quanto o adjetivo em posição pré-nominal, como em 'pa'ynha’u kamich' ("camisa nova”), indicando a possibilidade de movimento do adjetivo para uma posição mais alta, da mesma forma como ocorre em alguns casos do PB.

Evidentemente, a estrutura interna do DP em Wapichana necessita de mais estudos para que possamos apresentar uma estrutura estendida mais fina do DP nessa língua. Contudo, para os objetivos deste trabalho, foi possível verificar que, pelo fato de o Wapichana apresentar um sistema degenerado de determinantes, isto é, composto apenas por numeral, possessivo e demonstrativos, podemos aventar a hipótese de a estrutura expandida do DP ser composta pelos núcleos D, Num, Poss e N, com os demonstrativos ocupando o núcleo D. 
M.

Giovannetti

\& R. M. Basso

Demonstrativos,

determinantes

e definitude em

Wapichana

Contudo, encontramos também no Wapichana uma espécie de artigo ou, como propõe Giovannetti (2016), um processo de gramaticalização que está transformando um demonstrativo, a saber, 'taury'y', numa espécie de artigo. Os exemplos a seguir mostram o comportamento dos demonstrativos e de 'taury'y' em Wapichana, de modo que os demonstrativos propriamente ditos são compostos por 'diura'a', um demonstrativo distal, e 'wyry'y', um demonstrativo proximal:

\section{(1) Diura'a karich midi’u aquele livro grosso 'aquele livro é grosso'}
(2) Wyry'y kuraidiaunaa karina’u este criança doente adj 'Esta criança está doente'

A forma 'taury'y' é classificada por algumas gramáticas e trabalhos descritivos (cf. SANTOS, 2006) como um demonstrativo. Contudo, baseados em exemplos como (3), a seguir, argumentaremos contra essa análise, uma vez que tal sentença só é gramatical se 'taury’y' estiver ligado a um DP anterior na sentença ou no discurso; caso ele esteja sendo utilizado deiticamente, isto é, acompanhado de um gesto de ostensão, a sentença não é aceita pelos falantes como gramatical.

\section{(3) Taury'y daunaiura wautan Aquele homem cair \\ 'Aquele homem caiu'}

Para podermos verificar o status de 'taury'y', devemos primeiro definir quais características sintáticas e semânticas os demonstrativos possuem. Assim, na seção seguinte, apresentaremos alguns argumentos que podem melhor especificar a restrição morfológica existente no Wapichana em relação aos dois demonstrativos existentes e uma possível gramaticalização por que passa o item 'taury'y'.

\section{Características dos demonstrativos e da definitude}

Nesta seção, buscaremos apresentar o que se entende por demonstrativos e sua relação com a definitude nas línguas naturais. Partindo-se do entendimento de que, na maioria das línguas naturais, processos internos a elas são responsáveis por transformarem pronomes demonstrativos em artigos, e sendo estes os responsáveis em grande medida pela construção da definitude, apresentaremos algumas características comuns a esses itens pertencentes à classe que conhecemos como demonstrativos.

Revista Letras,

Curitiba, UFPR, n. 96, pp.423-441, jul./dez. 2017. ISSN 2236-0999 (versão eletrônica) 
As teorias existentes sobre demonstrativos nas línguas naturais, via de regra, tomam tais itens como dados, e não raro não fornecem ferramentas para a identificação desses itens, e o resultado é que não há grande clareza sobre o que exatamente são os demonstrativos; grosso modo, podemos dizer que demonstrativos são palavras usadas para mostrar algo saliente no contexto ou acompanhado por um gesto. Contudo, essa definição ainda não é satisfatória, uma vez que os artigos definidos, bem como diversos outros itens e construções, nas línguas naturais que os possuem, também podem ser usados para "mostrar algo" acompanhados de um apontamento.

Poderíamos, numa tentativa inicial, procurar definir os demonstrativos em termos de certos traços ou conjunto de traços específicos que os diferenciem, por exemplo, dos artigos; ou seja, ter em mãos um conjunto de elementos mínimos que determinariam o comportamento tanto dos demonstrativos quanto dos artigos nas línguas naturais. No entanto, é evidente que estes compartilham com aqueles uma grande quantidade de traços, haja vista que, diacronicamente, os artigos são derivados dos demonstrativos, segundo os estudos em gramática histórica.

De acordo com Diessel (1999), as características básicas apresentadas pelos demonstrativos são:

a) Demonstrativos são primariamente usados para focar a atenção do ouvinte em objetos ou lugares na situação de fala;

b) Eles são usados para organizar o fluxo de informação na fala em curso;

c) Demonstrativos são frequentemente usados para acompanhar os participantes de um discurso prévio e ativar o conhecimento específico compartilhado;

d) Sua função básica é orientar o ouvinte sobre a situação ao redor;

e) Normalmente eles são usados com uma demonstração.

Nossos dados mostram, entretanto, que 'taury'y' não possui nenhuma das características acima apresentadas, especialmente, pelo fato de não poder ser utilizado com uma demonstração.

'taury'y' não pode ser usado com uma demonstração ou gesto de apontamento, não apresentando, assim, as características em a), d) e e). Além disso, 'taury'y' não apresenta a oposição de traços semânticos dêiticos [+distal]/[-distal] 
M. e [+proximal]/[-proximal], como os itens 'diura'a' e 'wyry'y', caracterizados

GiovannetTi \& R. M. BAsSO

Demonstrativos, determinantes e definitude em Wapichana respectivamente como [+distal] e [+proximal].

Além dos traços [ \pm distal], [tproximal], os demonstrativos apresentam um traço semântico relacionado à definitude [ \pm def], e esse é justamente o caso de 'taury'y', um item que não pode ser usado com apontamento, não apresenta a distinção [+distal] e [+proximal], e apresenta o traço [ \pm def]. Dessa forma, argumentaremos que 'taury'y' está passando por um processo de gramaticalização, que o está direcionando a uma espécie de artigo definido em Wapichana, uma vez que não compartilha os traços semânticos apresentados por "diura'a" e "wyry'y", além de apresentar os traços semânticos característicos da definitude, de acordo com Roberts (2014), a saber:

- sentenças com 'taury'y', por este ser um item anafórico e dever estar ligado em sua categoria de regência, dependem de um referente antecedente na sentença, a fim de poder ser interpretado;

(4) Antônio tykypan [diaytan xuutunau $]_{\mathrm{i}}$ ixturu ii;

$\begin{array}{lll}\text { 3ps } & \text { ver dois camisa pl loja posp. } \\ \text { ikian } & \text { [taury'y xuutunau }]_{i} \text { kunaynamau } \\ \text { 3ps } & \text { dizer dem. camisa pl bonito pl }\end{array}$

'Antônio viu duas camisas na loja; ele disse que aquelas camisas eram bonitas' - por 'taury'y' ser sempre anafórico, uma sentença com este item em que ele não esteja ligado a um referente é anômala;

(5) *Taury’y daunaiura ma’uzka’u tykap nii kawaru kunaynamau

(em contexto de apontamento)

Dem menino forte ver n.p. cavalo bonito

'Aquele menino forte viu um cavalo bonito'

- deve haver alguma familiaridade informacional entre os interlocutores, isto é, os interlocutores devem compartilhar alguma informação a fim de satisfazerem a pressuposição de familiaridade. Neste caso, 'taury'y' funciona como um relativizador, introduzindo uma relativa restritiva, responsável pela familiaridade da informação compartilhada;

(6) Chapi'ik taury'y wichauraz

(informantes conversando sobre dias de verão vividos por ambos)

manhã dem quente

'As manhãs que eram quentes'2

${ }^{\star}$ Chapi'ik diura’a wichauraz

1 Embora tal construção se assemelhe muito às chamadas "small clauses livres", em discussão com os informantes, a melhor saída para glosa desse tipo de construção, em vários contextos, é como uma relativa.

2 A inferência de passado se dá contextualmente, pelo fato de os falantes estarem comentando sobre dias vividos por ambos em algum tempo do passado. Em Wapichana, os verbos de cópula não são realizados fonologicamente, exceto em alguns contextos muito específicos, e com

Revista Letras, alteração morfológica. Tal discussão foge do escopo deste trabalho.

Curitiba, UFPR,

n. 96, pp.423-441,

jul./dez. 2017. 


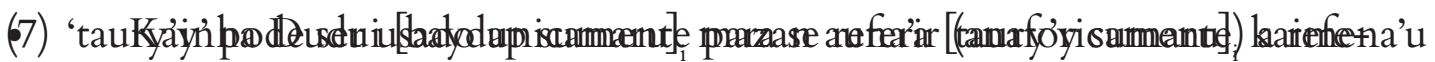

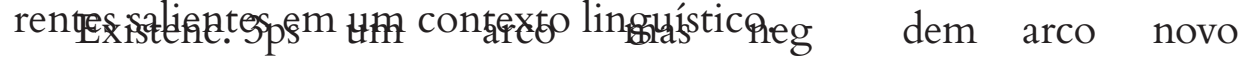

'Dudui tinha um arco, mas aquele arco não era novo'

`Kainha Dudui [baydap sumaru] ${ }_{\mathrm{i}}$ mazan auna’a [diura’a sumaru] $]_{\mathrm{i}}$ kaimena’u

Podemos observar, pelos exemplos de (4) e (7), que 'taury'y' aparece como um item anafórico no contexto linguístico em que aparece, retomando o DP "sumaru", tornando todo o complexo "taury'y sumaru" correferente ao complexo "baydap sumaru”. O mesmo não ocorre com 'diura’a', que não funciona como um item anafórico, retomando o DP correferente "baydap sumaru", uma vez que 'diura'a' funciona exclusivamente em contextos demonstrativos.

As características apresentadas por Roberts (2014) são basicamente as características de casos de anáfora. Portanto, espera-se que, em casos mais comuns, o termo anafórico e o referente retomado devem compartilhar alguns traços, mais conhecidos como traços- $\Phi$, isto é, traços referentes a pessoa, número e gênero, presentes nos nomes e determinantes nas línguas naturais. Aparentemente, o item 'diura'a', no Wapichana, não apresenta traços de número, pessoa e gênero, podendo ser usado como demonstrativo em toda e qualquer situação; o mesmo ocorre com o item 'taury'y', que pode retomar anaforicamente seres, objetos, eventos etc. No Wapichana, há uma complementariedade entre os itens 'diura'a' e 'taury'y', de modo que a mesma forma fonológica não pode ser usada tanto como demonstrativo quanto como elemento anafórico, e há assim especialização de um único elemento para determinada função, diferentemente do PB ('este', 'aquele'), do inglês ('this', 'that'), do italiano ('questo', 'quello') entre outra línguas, em que demonstrativos são também anafóricos.

$\mathrm{Na}$ seção abaixo, exploraremos melhor a ideia de gramaticalização, explicitando os conceitos relevantes e seu papel na explicação dos nossos dados.

\section{Gramaticalização: alguns critérios}

Nesta seção, apresentaremos brevemente uma discussão sobre gramaticalização. Para tanto, discutiremos a origem desse conceito nos estudos linguísticos, apresentando de modo geral o que podemos entender por tal processo. Em seguida, passaremos a discutir o processo de gramaticalização por um viés formal, relacionado à parametrização a que cada língua está sujeita.

A noção de gramaticalização foi introduzida nos estudos linguísticos por Meillet (1912) com o objetivo de designar um certo tipo de fenômeno linguístico 
M.

GiovannetTi

\& R. M. Basso

Demonstrativos, determinantes e definitude em Wapichana

de natureza diacrônica. O fenômeno em questão era a "transição gradual" que ocorria nas palavras ditas "principais", tornando-as palavras "acessórias", em seguida, num contínuo, essas palavras passariam a palavras "gramaticais"; esse processo seria observado ao longo dos vários estágios por que passa toda e qualquer língua.

Segundo Hopper e Traugott (1993, p. 7), apud Vital e Ramos (2006, p. 19-20), o processo de gramaticalização obedece a uma ordem fixa, passando sempre de uma estrutura lexical plena para uma estrutura gramatical plena, seus dois pontos limites. Uma estrutura lexical plena seria o que morfologicamente consideramos com "palavra" (cf. ARONOFF \& FUDEMAN, 2010.), ou seja, grosso modo, sintaticamente uma palavra é a menor unidade linguística que pode ocorrer sozinha, por exemplo, como resposta a uma pergunta. Exceto em casos de perguntas muito específicas, como as de cunho metalinguístico ou epilinguístico, uma palavra gramatical não poderia ser considerada, de fato, palavra por esse mesmo critério. Seria muito fácil para qualquer um, ao ser questionado sobre o que é um 'livro', apontar para um objeto no mundo; contudo, tal facilidade se esvairia se se questionasse sobre o que é um 'que' ou um 'o'.

Não obstante, na história das línguas, é comum encontrarmos casos em que itens pertencentes às categorias lexicais passam a fazer parte das categorias gramaticais, como podemos observar no caso dos verbos auxiliares, algumas preposições - as chamadas funcionais -, transformando-se, no limite, em clíticos e afixos. Além dessas classes, uma que encontramos frequentemente nas línguas que possuem artigos definidos é a passagem de demonstrativos, considerados em um ponto da sincronia da língua, a artigos.

Ainda segundo Vitral e Ramos (op. cit.), há alguns eixos de estudos possíveis acerca do processo de gramaticalização; dentre eles, podemos encontrar aqueles ligados às ideias pioneiras de Humboldt, que enfatiza a chamada "evolução das categorias da língua”, focando os processos diacrônicos que são reanalisam os itens lexicais em itens gramaticais, como por exemplo, a passagem de verbo pleno a verbo auxiliar.

Outro modo de enxergar a gramaticalização, apresentado pelos autores, é colocar em destaque a interação entre a sintaxe e o discurso, de modo que os padrões sintáticos apresentados são resultantes do próprio uso linguístico, indo contra a autonomia do componente gramatical da faculdade da linguagem. Seriam as exigências comunicativas que gerariam as regularidades observadas no nível oracional, como nos mostram os trabalhos de Sankoff e Brown (1976) e Givón (1979) apud Vitral e Ramos (op. cit.).

Como podemos observar até aqui, grande parte dos trabalhos sobre gramaticalização possui um viés funcionalista; disso, surge uma questão importante: como conciliar gramaticalização com os estudos de linguística formal? Para que haja tal conciliação, faz-se necessária uma reinterpretação dos pressupostos teóricos para o modelo formal; o modelo que tomaremos aqui é o da

Revista Letras,

Curitiba, UFPr, n. 96, pp.423-441,

jul./dez. 2017. ISSN 2236-0999 (versão eletrônica) 
gramática gerativa em sua fase mais recente, o Programa Minimalista. Segundo Hopper e Traugott (op.cit.), quando um item se gramaticaliza, as seguintes etapas devem ser obedecidas:

(8) item lexical $>$ b. item gramatical $>$ c. clítico $>$ d. afixo

Observa-se que, durante esse processo de gramaticalização, a primeira etapa a ocorrer é a alteração da categoria a que a palavra pertence; isto é, deixamos de ter uma palavra com conteúdo lexical e passamos a ter uma palavra com um esvaziamento semântico. Em seguida, começam a ocorrer processos morfofonológicos, responsáveis pela alteração da estrutura da palavra.

Esse processo pode ser visto ao observarmos o processo de gramaticalização que ocorreu com o verbo auxiliar latino 'habere' transformando-se em um morfema de tempo futuro, como observamos a seguir:

(9) amare habeo > amare a(b)eo > amare *aio > amare *eio > amare ei (amarei)

No PB, uma das formas de verificarmos a gramaticalização ocorrida do verbo auxiliar 'habere' é a ocorrência da mesóclise. No inglês moderno, também vemos um processo de gramaticalização no curso, qual seja, a formação do futuro com 'going to'; é cada vez mais corrente, ao menos na língua falada, a passagem da forma 'going to' para a forma reduzida 'gonna', havendo, primeiramente, perda da carga semântica do verbo 'go' (ir) e consequente perda de material fonológico.

Partindo-se de um viés gerativo, o processo de gramaticalização deve ser reinterpretado a partir de uma mudança nos parâmetros apresentados por uma determinada língua, fazendo com que determinado item se gramaticalize.

É importante notarmos que o esquema de Hopper e Traugott acima apresentado não implica que todo processo necessite obrigatoriamente apresentar todas aquelas etapas; ou seja, como no caso dos verbos auxiliares, não encontramos mudanças ocorridas em seu aspecto morfofonológico.

Vale notarmos, também, que a gramaticalização envolve processos em que um item lexical é recategorizado como gramatical e processos em que não ocorre essa recategorização, de modo que esse item permanece na mesma categoria que outrora. Os fenômenos do primeiro tipo podem ser exemplificados com a passagem dos demonstrativos a artigos; os do segundo, com o fenômeno semântico da metáfora, em que um item se especializa semanticamente, como no caso de "braço da cadeira", "asa da xícara", etc.

Não obstante, o processo de gramaticalização propriamente dito ocorre sempre quando um item pertencente a uma das classes lexicais passa a funcionar como um item de uma das classes gramaticais. Dessa forma, o viés gerativo pode auxiliar na compreensão do processo de gramaticalização na medida que permite explicitar o comportamento sintático dos itens, oferecendo uma 
M. justificativa teórica de tal comportamento em termos das hierarquias estruturais

GiovanNETTI \& R. M. BASSO

Demonstrativos, determinantes e definitude em Wapichana apresentadas.

A seguir, analisaremos a estrutura funcional do DP em Wapichana e, na seção 5 , mostraremos o processo de gramaticalização por que passa o item 'taury'y'.

\section{Gramaticalização do demonstrativo 'taury'y'}

$\mathrm{Na}$ maior parte das línguas naturais, os demonstrativos compartilham entre si os traços apresentados por Diessel (1999), na seção 3, enquanto os anafóricos compartilham os traços apresentados por Roberts (2014), na mesma seção. Tanto demonstrativos quanto anafóricos apresentam a mesma distribuição sintática na maioria das línguas naturais, além de apresentarem a mesma morfologia, como podemos observar no PB, com as formas "este", "esse" e "aquele" (anafóricos e demonstrativos), no inglês, com as formas "this" e "that" (anafóricos e demonstrativos) - além do fato de "that" ocupar o núcleo C, o que nos leva a questionar o fato de estarmos diante de formas homonímicas, em que o demonstrativo tenha se gramaticalizado em complementizador, sem sofrer alterações morfofonológicas. É interessante notarmos que, no inglês, o processo de gramaticalização que deu origem ao artigo definido 'the' teve início como o demonstrativo 'that'; seguindo os processos apresentados acima (HOPPER; TRAUGOTT, 1993), temos a passagem de um item lexical 'that' para um item gramatical 'the' com a ocorrência de alteração morfofonética. Vale observarmos que os autores apontam para essa alteração apenas quando da passagem de itens gramaticais a clíticos e afixos; no entanto, observamos tal alteração na passagem de 'that' a 'the', no inglês, e de 'ille', no latim, a 'o', no PB.

Alguns trabalhos sobre a língua Wapichana, como Santos (2006), atribuem ao item 'taury'y' um status de demonstrativo, como podemos ver nos exemplos a seguir (SANTOS, 2006, p. 188):

(10) taw-ru zin maku-n zakap it

DIST $f$ mulher ir-MI roça ALL

'aquela mulher vai para a roça'

(11) tawi-ri dauniur maku-n zakap it

DIST $m$ homem ir-MI roça ALL

'aquele homem vai para a roça'

(12) tawiri kuti?iz, zįitit-p-a-n

DIST pássaro voar-CONT-EP-MI

'aquele pássaro está voando'

(13) ũ-aiap tawiri baru

1-precisar DIST machado

'eu preciso daquele machado'

Revista Letras,

Curitiba, UFPR,

n. 96, pp.423-441,

jul./dez. 2017.

ISSN 2236-0999

(versão eletrônica) 
Contudo, em novos trabalhos de elicitação realizados, verificou-se que o item 'taury'y' não aparece em contextos de demonstração, mas apenas em contextos anafóricos, em que há um constituinte sendo retomado ou sintaticamente ou pragmaticamente.

Os exemplos a seguir nos mostram que 'taury'y' não pode ser usado em contextos de demonstração, enquanto 'diura'a' não pode sê-lo em contextos anafóricos:

i) 'taury'y' não é permitido com demonstração, ou seja, ele precisa valorar traço [+anaf] no contexto linguístico em que ocorre

(14) `Taury'y daunaiura ma’uzka’u tykap nii kawaru kunaynma’u (contexto demonstrativo)

Aquele menino força adj ver np cavalo beleza adj 'Aquele menino forte viu um belo cavalo'

Como o DP ‘taury’y daunaiura' não está ligado a nenhum outro DP na sentença, 'taury'y' não pode ser usado em contexto de demonstração, e a sentença torna-se estranha, uma vez que o referente de "taury'y daunaiura” não consegue ser atingido. Contudo, quando há um NP com o qual possa haver a relação anafórica, a sentença torna-se gramatical com a forma 'taury'y':

ii) Correferencial com um NP precedente

(15) Pedro tyka'ap nii [bauran zinabe] dunui ii ykien [taury'y zinabe $]_{i}$ kunanma'u manawyn Maria ai

'Pedro viu outra garota na cidade e ele disse que aquela garota era mais bonita que Maria'

O DP 'taury’y zinabe' possui uma relação anafórica com o DP 'bauran zinabe', seu referente sintático; devemos observar que se a forma utilizada fosse 'diura’a', não haveria a formação de anáfora, e a sentença seria agramatical. 
M.

GiovannetTI

\& R. M. Basso

Demonstrativos, determinantes e definitude em Wapichana iii) Bridging

(16) Wamakunkan Mariwyd ii baukuptimpkizei waabata'anan mazan (taury'y) imzuwaynaa kawan ydayna'an zii

Wa maku- -nka -n Mariwyd ii baukuptimpkizei wa- abata'anan mazam (taury'y)

1pp ir ? MI Pium em reunião 1pp ouvir mas dem.

imzuwaynaa kawan y- dayna’an zii

tuxaua chegar 3ps ainda

'Nós estivemos na reunião no Pium, mas o tuxaua (i.e. o chefe) estava atrasado'

O fenômeno de bridging, às vezes chamado de "anáfora associativa", se dá quando um elemento anafórico é usado sem a presença explícita de seu antecedente, mas sim de um nome que licencia uma inferência anafórica. Considere a sentença “Ontem, fomos a uma exposição, e os quadros eram lindos”; a descrição 'os quadros' é anafórica, e seu antecedente seria algo como "os quadros da exposição a que fomos ontem", que não é dado explicitamente. Daí a ideia de "associação" ou "ponte" (i.e. bridging) entre um nome e um antecedente não explicitado, mas recuperado contextualmente. No exemplo em (16), "reunião no Pium" licencia o uso da descrição 'o tuxaua' (na glosa), configurando um caso de bridging.

iv) Homônimo como relativizador ${ }^{3}$

(17) Chapiiłk taury'y wichauraz manhã dem quente

'As manhãs que eram quentes'

Vale ressaltarmos que, no Wapichana, a ordem no interior do DP é fixa [Dem DP], o que impediria a interpretação de 'taury'y' em (17) como um demonstrativo com escopo sobre o DP 'chapi'ik'; assim, 'taury'y' não formaria um constituinte com o DP 'chapi'ik', mas introduziria uma relativa restritiva, como podemos observar em (18):

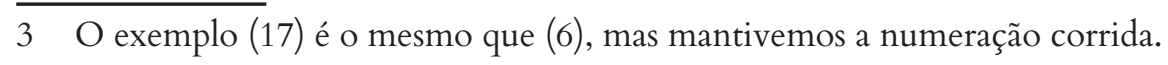

Revista Letras,

Curitiba, UFPR, n. 96, pp.423-441, jul./dez. 2017. ISSN 2236-0999 (versão eletrônica) 
(18) Ipei zynaa taury'y kunaikiauraz naydapnii pakanaichan ${ }^{4}$ toda menina que feliz gostar np poss cantar prog.

'Toda menina que é feliz gosta de cantar'

Nessas sentenças acima, podemos verificar que 'taury'y' ocupa [Spec, CP], comportando-se como um relativizador, retomando o DP antecedente 'zynaa' em (18) e o DP ‘chapi'ik' em (17). O demonstrativo 'diura'a' não poderia ocupar Spec, CP, uma vez que não pode ser usado como relativizador, logo, a sentença seria semanticamente agramatical, como observamos em (19a):

(19) a. *Ipei zynaa diura’a kunaikiauraz naydapnii pakanaichan

b. Wyry'y zynaa taury'y diura'a daunaiura tykapnii essa menina que aquele menino ver $\mathrm{np}$ naydapnii pakanaichan

gostar np poss cantar prog

'Essa menina que aquele menino viu gosta de cantar'

Em (19b) observamos a coocorrência de 'diura'a' e 'taury'y' em "taury'y diura'a daunaiura”; ora, se ambos estivessem sendo usados como demonstrativos/ anafóricos, a sentença seria agramatical, uma vez que concorreriam pela mesma posição na sentença, a saber, o núcleo determinante.

De fato, o que ocorre é que temos 'taury'y' como um relativizador, ocupando [Spec, CP] e 'diura'a' como demonstrativo, ocupando a posição de determinante no DP. Assim, por apresentarem distribuições distintas na sentença, a coocorrência de ambos não gera uma sentença agramatical.

O fato de alguns falantes tratarem tanto 'taury'y' quanto 'diura'a' como demonstrativos, inclusive sendo essa a descrição presente em trabalhos prévios sobre a língua (como a Gramática Pedagógica do Wapichana - a ser lançada pelo Museu do Índio -, e o trabalho descritivo de Santos, 2006), bem como os dados elicitados nas comunidades Malacacheta e Pium, permite concluir que, em algum momento da diacronia da língua Wapichana, o item 'taury'y' se comportou como uma forma lexical, um demonstrativo, como podemos observar nos exemplos de (10) a (13); contudo, no estágio atual da língua, segundo o resultado de nossas elicitações, estamos verificando um processo de gramaticalização desse item, que o está levando a se comportar como uma espécie de artigo definido, além da sua forma homônima como relativizador, como vemos em (17) e (18).

4 Um dos pareceristas sugeriu que 'taury'y' fosse um demonstrativo realizado à direita do núcleo nominal. Contudo, como discutido na seção 2, a estrutura do DP em Wapichana obedece a uma ordem fixa [Det DP], de modo que 'taury'y' tem a mesma distribuição que 'ipei'; dessa forma, uma construção como 'Ipei taury'y zynaa' é agramatical, segundo os falantes. 
M.

GiovanNETTI

\& R. M. BAsSo

Demonstrativos,

determinantes

e definitude em

Wapichana

\section{Conclusão}

Este trabalho foi um exercício teórico-analítico na tentativa de verificar em que medida a distinção 'demonstrativo'/‘anafórico', em Wapichana, pode ser definida em termos de processos de gramaticalização. Discutimos alguns aspectos tipológicos do Wapichana, nos focando principalmente na estrutura interna do DP, mostrando a existência de demonstrativos que ocupam um núcleo superior ao NP, a saber, 'diura'a' e 'wyry'y'; no entanto, apontamos para a necessidade de mais pesquisas sobre a estrutura interna do DP naquela língua.

$\mathrm{Na}$ sequência, lidamos com o comportamento de uma outra forma existente na língua e tratada como demonstrativo, a forma 'taury'y', embora não apresente nenhuma característica formal pertencente àquela classe. Para tanto, apresentamos traços que caracterizam a classe dos demonstrativos, segundo Diessel (1999), e verificamos que ‘taury'y' não apresenta nenhum traço que possa caracterizá-lo como tal, embora apresente algumas características de definitude, trazidas por Roberts (2014), o que nos leva a manter nossa hipótese de que 'taury'y' apresenta mais traços ligados a traços apresentados pelos artigos definidos que traços apresentados pelos demonstrativos.

Pudemos verificar também que 'taury'y' apresenta características semelhantes às dos pronomes relativos, podendo aparecer como pronome relativo em algumas construções, o que pode corroborar nossa hipótese de gramaticalização em processo na língua Wapichana. Não obstante, no estágio atual em que se encontra a língua, não podemos asseverar que o processo de gramaticalização de fato se efetivará, produzindo um artigo definido naquela língua, bem como se temos uma mesma palavra que está num estágio de gramaticalização que a transformou numa espécie de relativizador ou se se trata de homônimos, cada um com seus respectivos comportamentos sintático e semântico. 


\section{Referências}

ABNEY, S. The English noun phrase in its sentential aspects. Tese de doutorado, MIT, 1987.

AIKHENVALD, A. Y. The Arawak language family. In: DIXON, R. M. W.; AIKHENALD, A. Y. (Eds.) The Amazonian Languages. Cambridge: Cambridge University Press, 1999.

ARONOFF, M.; FUDEMAN, K. What is morphology? 2nd Edition. Chichester, UK: Wiley-Blackwell, 2010.

BRUGÈ, L. The positions of demonstratives in the extended nominal projection. In: CINQUE, G. (Ed). The cartography of syntactic structures. Vol. 1. Oxford: Oxford University Press, 2002. pp. 15-53.

CINQUE, G. The functional structure in DP and IP. Vol. 1. Oxford: Oxford University Press, 2002.

DIESSEL, H. The morphosyntax of demonstratives in synchrony and diachrony. Linguistic typology, v. 3, p. 1-49, 1999.

GIOVANNETTI, M. Notes on demonstratives and anaphorics in Wapishana. Presentation in "Workshop Pronouns: Morphosyntax, Semantics and Processing”, Universidade Federal da Bahia, Salvador, 2016.

GIUSTI, G. The functional structure of noun phrase: a bare phrase structure approach. In: CINQUE, G. (Ed). The cartography of syntactic structures. Vol.1. Oxford: Oxford University Press, 2002. pp. 54-90.

GIVÓN, T. Toward a Discourse Definition Syntax. Unpublished MS. University of California at Los Angeles, 1974.

GREENBERG, J. The general classification of Central and South American Languages. Selected papers of the 5th International Congress of Anthropological and Ethnological Sciences. Philadelphia, 1956.

HOPPER, P.; TRAUGOTT, E. Grammaticalization. Cambridge: Cambridge University Press, 1993.

MEILLET, A. Linguistique historique et linguistique générale. Paris: Edouard Champion, 1948 [1912].

MIGLIAZZA, E. C. Languages of the Orinoco-Amazon Region: current status. In: KLEIN, H. E. M.; STARK, L. R. (Eds.) South American Indian Languages retrospect and prospect. Austin: University of Texas Press, 1985. p. 286-303.

PAYNE, D. L. A classification of Maipuran (Arawakan) languages based on shared lexical retentions. In: DERBYSHIRE, D. C.; PULLUM, G. K.. (Eds.). 
M. Handbook of Amazonian languages. Berlin/New York: Mouton - De Gruyter,

GiovannetTi $\&$ R. M. BAsSO

Demonstrativos, determinantes e definitude em Wapichana 1991. v. 3, p. 355-499.

PIRES DE OLIVEIRA, R. GIOVANNETTI, M. The nominal system in Wapishana (Aruak): first thought. In. https:/www.researchgate.net/ publication/310803343_The_nominal_system_in_Wapishana_Aruak_first_ thought

ROBERTS, C. Indexicality: de se semantics and pragmatics, Manuscript. 2014.

RODRIGUES, A. D. Línguas brasileiras - para o conhecimento das línguas indígenas. São Paulo: Loyola, 1986.

RUSSELL, B. On denoting. Mind (New Series), v. 14, n. 56, p. 479- 493, out. 1905.

SANKOFF, G.; BROWN, P. The origins of syntax in discourse: a case study of Tok Pisin relatives. Language, v. 52, n. 3, p. 631-667, 1976.

SANTOS, M. G. Uma gramática do Wapixana (Aruák): aspectos da fonologia, da morfologia e da sintaxe. Tese (Doutorado em Linguística). Universidade Estadual de Campinas, Campinas, 2006.

SCOTT, G. -J. Stacked adjectival modification and the structure of nominal phrases. In: CINQUE, G. (Ed.) The cartography of syntactic structures, vol.1. Oxford: Oxford University Press, 2002. pp. 91-122.

VITRAL, L.; RAMOS, J. Gramaticalização: uma abordagem formal. Rio de Janeiro: Tempo Brasileiro; Belo Horizonte: Faculdade de Letras FALE/ UFMG, 2006.

VON DEN STEINEN, K. Entre os aborígenes do Brasil Central. São Paulo: Departamento de Cultura, 1940. Edição original: 1886.

Submetido em: 21-02-2017

Aceito em: 06-06-2017

Revista Letras, Curitiba, UFPR, n. 96, pp.423-441, jul./dez. 2017. ISSN 2236-0999 (versão eletrônica) 